\title{
Electron Localization and a Confined Electron Gas in Nanoporous Inorganic Electrides
}

\author{
Peter V. Sushko, ${ }^{1, *}$ Alexander L. Shluger, ${ }^{1}$ Katsuro Hayashi, ${ }^{2}$ Masahiro Hirano, ${ }^{2}$ and Hideo Hosono ${ }^{2}$ \\ ${ }^{1}$ Department of Physics and Astronomy, University College London, Gower Street, London WCIE 6BT, United Kingdom \\ ${ }^{2}$ Transparent Electro-Active Materials Project, Exploratory Research for Advanced Technology (ERATO), Japan Science and \\ Technology Corporation, KSP C-1232, 3-2-1 Sakado, Takatsu-ku, Kawasaki 213-0012, Japan
}

(Received 1 April 2003; published 18 September 2003)

\begin{abstract}
The nanoporous main group oxide $12 \mathrm{CaO} \cdot 7 \mathrm{Al}_{2} \mathrm{O}_{3}(\mathrm{C} 12 \mathrm{~A} 7)$ can be transformed from a wide-gap insulator to an electride where electrons substitute anions in cages constituting a positive frame. Our $a b$ initio calculations of the electronic structure of this novel material give a consistent explanation of its high conductivity and optical properties. They show that the electrons confined in the inert positive frame are localized in cages and undergo hopping between neighboring cages. The results are useful for the understanding of behavior of confined electron gas of different topology and electron-phonon coupling, and for designing new transparent conductors, electron emitters, and electrides.
\end{abstract}

DOI: 10.1103/PhysRevLett.91.126401

PACS numbers: 71.55.Ht, 61.72.Bb, 61.72.Ji, 71.20.-b

The recent advent of inorganic electrides, crystalline salts where electrons serve as the anions, opens exciting opportunities for studying the properties of an electron gas of controlled density confined in regular nanopore arrays over a wide temperature range. In electrides based on silica zeolites doped with Cs [1], the low-density electron gas is confined within the cavities and channels of the original structure, and between $\mathrm{Cs}^{+}$ions arranged in short-range ordered zigzag chains providing charge neutrality [2]. Another material exhibiting similar properties is a nanoporous main group oxide $12 \mathrm{CaO} \cdot 7 \mathrm{Al}_{2} \mathrm{O}_{3}$ (C12A7) [3]. It is composed of a positively charged framework built from 12 cages and of two extraframework $\mathrm{O}^{2-}$ ions occupying two different cages, and can be represented by a chemical formula $\left[\mathrm{Ca}_{24} \mathrm{Al}_{28} \mathrm{O}_{64}\right]^{4+} \cdot 2 \mathrm{O}^{2-}$. After special treatment [3,4], the interstitial oxygen ions are substituted by electrons confined in inert lattice cages. The concentration of thus formed electron gas can be varied between about $10^{19}$ to $2 \times 10^{21}$ electrons [4] exhibiting a spectrum of different optical and magnetic behaviors. At the dilute limit, the electrons are thought to be localized and undergo variable range hopping at low temperatures [3]. At the high concentration limit, the electrons tend to pair and demonstrate antiferromagnetism [4]. These materials are transparent conductors and powerful reducing agents. Studies of these novel and poorly understood crystals provide a deeper insight into the dependence of electron gas behavior on dimensionality and topology, and into design of new transparent conductor, electron emitters, and electrides.

This Letter presents results of the first electronic structure calculations for an inorganic electride. Calculations of even relatively better-known organic electrides are very scarce because of their complexity [5]. We focus on the dilute extra electron limit, where more experimental data are available. Pure C12A7 samples are optically transparent up to $6.0 \mathrm{eV}$. However, after loading with hydrogen and then irradiating with UV light, single crystals acquire high electrical conductivity due to formation of a significant number of unpaired electrons, as do sintered powders and thin films. This conductivity is accompanied by formation of two broad optical absorption bands with maxima at about 0.4 and $2.8 \mathrm{eV}$. It has been suggested [3] that similar to positively charged oxygen vacancies in many oxides, positive cages forming the frame of this material may serve as trapping centers for at least one electron. However, in C12A7 cages constitute the crystal lattice, whereas vacancies are located a long distance apart. Therefore the electron localization is remarkable and may depend on the electron gas concentration. The results of $a b$ initio calculations described in this paper demonstrate that the cage electrons are coupled to a strong lattice deformation and provide a consistent explanation for conductivity and optical absorption in this system.

The $\mathrm{C} 12 \mathrm{~A} 7$ is a cubic crystal, which belongs to the I-43d space group and has the lattice constant of $11.99 \AA$ [6]. The 118 atom unit cell contains two C12A7 molecules and is composed of cages shown in Fig. 1. The cages are approximately $6 \AA$ wide and connected to eight other cages via $3.5 \AA$ wide openings (see Figs. 1 and 2 ). An effective positive charge of each cage $(+1 / 3 e)$ is a peculiar property of this system, which differs from cage sodalites (see, for example, Ref. [7]) and other electrides [8]. To study properties of unpaired electrons in C12A7, we employed an embedded cluster technique, which allows one to calculate optical, paramagnetic, and transport properties of individual defects. A cluster with a defect treated quantum mechanically is embedded in the rest of the polarizable crystal treated classically (see Figs. 1 and 2). This allows one to account for both ionic and electronic contributions to the polarization of the defect environment. This technique has been thoroughly described in Refs. [9,10] and tested in defect studies of several oxides, such as $\mathrm{MgO}$ [9] and $\mathrm{SiO}_{2}$ [10]. 


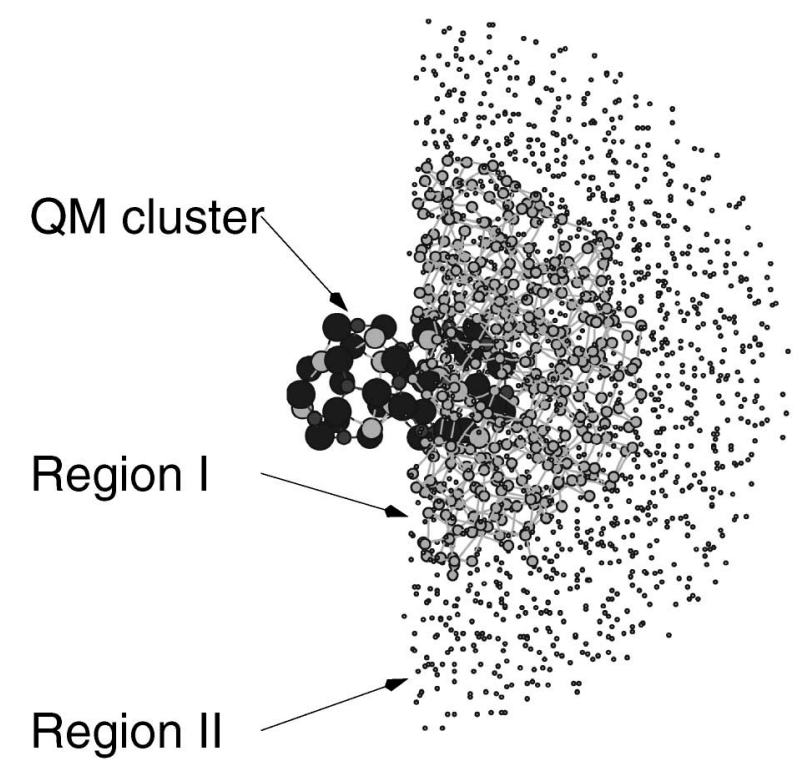

FIG. 1. The setup of embedded cluster calculation: The quantum cluster including two cages is in the middle surrounded by regions I and II of classical ions. Only half of the classical ions are shown for clarity.

A crystal with a defect is represented by a large finite nanocluster of nearly spherical shape built from 64 unit cells and divided into regions I and II as briefly described below (see Fig. 1). Two adjacent cages placed at the center of the nanocluster are treated quantum mechanically (QM cluster) and surrounded by a spherical region I with the radius of about $13 \AA$. The remaining part of the nanocluster, region II, is represented by classical nonpolarizable ions. Ions outside the QM cluster are treated using the classical shell model and interatomic potentials [11]. All centers in region I, containing about 700 atoms, are fully relaxed in the course of energy minimization while those in the rest of the nanocluster are kept fixed in perfect lattice positions. All oxygen ions in the QM cluster are represented as all-electron atoms with the standard 6-31G basis set. The $\mathrm{Ca}$ and $\mathrm{Al}$ ions are treated using the lan12 effective core pseudopotential (ECP) and the lanl2dz basis set [12]. To provide a better flexibility, an additional basis obtained by extending the standard 6-311G* basis set for oxygen atoms by two $s p$ functions was positioned at the center of each of the two QM cages. The interface between the QM cluster and classical ions is introduced to prevent an artificial spreading of electronic states outside the QM cluster. $\mathrm{Ca}$ and $\mathrm{Al}$ ions in the interface region are represented using ECPs and interact quantum mechanically with atoms of the QM cluster and classically with the classical environment.

The effective charges of oxygen ions fully coordinated by quantum ions are very similar to those in the QM cluster wall bordering the classical region. The maximum difference in effective charges on $\mathrm{Ca}$ and $\mathrm{Al}$ ions within the QM cluster does not exceed $0.3 e$. The equiva- lent $\mathrm{Ca}-\mathrm{O}$ and $\mathrm{Al}-\mathrm{O}$ distances within the $\mathrm{QM}$ cluster do not differ by more than $0.04 \AA$.

This technique is implemented in a computer code GUESS $[9,10]$ (Gaussians used for embedded system studies), which interfaces the GAUSSIAN98 package [13] for quantum mechanical calculations with a program for classical calculations using the shell model available within the GUESS code. Calculations were made using density functional theory (DFT) with a hybrid density functional B3LYP and the optical transition energies were calculated using the time-dependent DFT method (TD-DFT) as implemented in GAUSSIAN98 [13].

Extra-framework oxygen ions tend to minimize their electrostatic repulsion but do not form an ordered sublattice. Among oxygen configuration, we have chosen one that corresponds to the smallest dipole moment per crystal unit cell. An extra-framework oxygen ion in the QM cluster was substituted by one or two electrons. The two cages of the QM cluster are not equivalent: One of them (cage 1) has a classical neighboring cage with an $\mathrm{O}^{2-}$ in it, whereas the other one (cage 2) does not have an extra-framework oxygen ion in the neighboring cages. Calculations demonstrate that two electrons prefer to delocalize over the two cages, so that each cage contains one electron, and interact only weakly. Therefore, in the following discussion, we focus on a single electron case.

The results of calculations demonstrate that the extra electron can be localized in either of the cages (see Fig. 2). The electron density has a predominantly $s$ character and is localized at the center of a cage in the same fashion as a single electron is localized in an anion vacancy of, e.g., $\mathrm{MgO}$ [9]. The isosurface of the one-electron function associated with the cage electron in Fig. 2 and the integration of the electron density show that most of the electron density (about 85\%) is confined within one cage, while the other one remains almost empty. Similar electronic structure was obtained for the electron localized in the other cage. The calculated hyperfine interaction of the

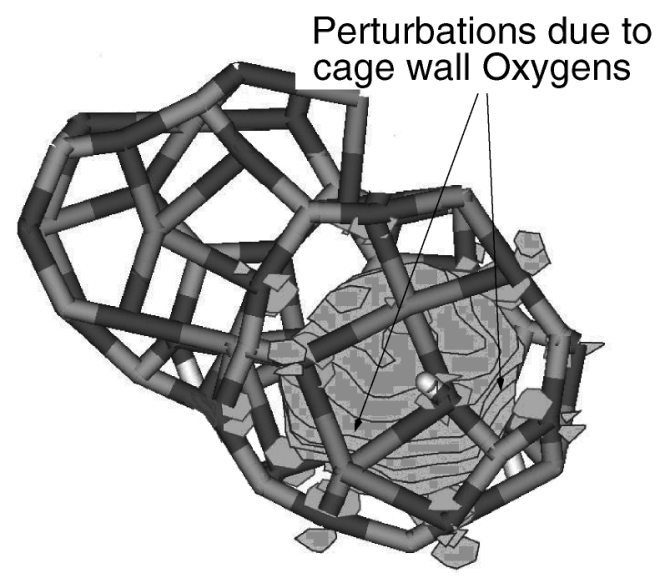

FIG. 2. The isosurface of the wave function of the electron localized in one of the $\mathrm{C} 12 \mathrm{~A} 7$ cages. 
unpaired electron with the nuclei of the neighboring $\mathrm{Al}$ ions is quite small and does not exceed $0.5 \mathrm{mT}$. This is mainly due to the fact that $\mathrm{Al}$ ions are screened by the surrounding oxygen ions, which repel the extra electron (see Fig. 2). The calculated hyperfine interaction with oxygen ions in the cage wall is about $1 \mathrm{mT}$.

Comparison of the local atomic structures obtained for the electron localized in two different cages reveals a characteristic lattice distortion, with the strongest relaxation of two $\mathrm{Ca}$ ions in each cage located on the opposite sides of the wall with the center of the cage approximately between them. Unlike other $\mathrm{Ca}$ and $\mathrm{Al}$ ions, they are exposed to the electron density localized in the cage and displace towards its center by about $0.4 \AA$. Most of the other ions displace away from the center and their relaxation propagates up to $10 \AA$ from the cage center. These large displacements reflect the strong electronphonon interaction due to elasticity of the cage walls. The corresponding soft phonon modes are discussed in a separate paper [14].

The number of variables involved in the geometry optimization in our calculations exceeds two thousand, which makes the search for an adiabatic barrier for the electron hopping from cage 1 to cage 2 extremely computationally demanding. Therefore we used a simpler linear interpolation approach. Let $\mathbf{R}_{1}$ and $\mathbf{R}_{2}$ be coordinates of all atoms for the two configurations of the electron localized in cage 1 and cage 2 , respectively. Then the coordinates of all the lattice atoms along the hopping path can be approximated as $\mathbf{R}=t \times \mathbf{R}_{1}+(1-t) \times \mathbf{R}_{2}$, where $t$ is a parameter varied in the range from 0 to 1 . This allows us to calculate all points along the adiabatic path with the same accuracy but the calculated barrier represents the upper limit for the "true" hopping barrier. We obtain a very small barrier of about $0.1 \mathrm{eV}$ for the hopping from cage 1 to cage 2 , and $0.17 \mathrm{eV}$ for the hopping from cage 2 to cage 1 .

We have calculated the optical absorption energies and the corresponding transition matrix elements for the lowest excited state, $E_{1}$, along the hopping path using TD-DFT. The vertical transition energy in the minima is about $1 \mathrm{eV}$ with a relatively small transition matrix element. If the system is displaced from an equilibrium position in either cage towards the barrier point, $E_{1}(t)$ decreases to about $0.6 \mathrm{eV}$. At the same time, the transition matrix element increases, indicating a higher transition probability. The highest occupied and lowest unoccupied one-electron molecular orbitals (HOMO and LUMO, respectively) are related to each other in the following way. If the system is in one of its two fully relaxed ground state configurations, say $t=1$, then the HOMO is fully localized in cage 1 and has the $s$ character as discussed above. The corresponding LUMO has two components: Its predominantly $s$-character maximum is localized in cage 2 with a smaller $p$-character contribution extended to cage 1. The reverse applies to the HOMO and LUMO calculated for the parameter $t=0$. Thus, the lowest optical excitation corresponds to the electron transfer between cages (see Fig. 3). When the system is displaced from the minimum towards the barrier, the HOMO attains two weakly overlapping $s$-type components localized in cages 1 and 2 with the ratio between their maxima determined by $t$; the LUMO has two $s$-type components of different sign localized in the two cages with the ratio between their maxima reverse with respect to that found for HOMO. This leads to a strong increase in the transition matrix element close to the saddle point. In the saddle point, the HOMO and LUMO can be qualitatively described as a symmetric and antisymmetric linear combination of $s$-type functions localized within the two cages: $s_{1}+s_{2}$ and $s_{1}-s_{2}$, respectively.

The optical transitions into three higher excited states calculated using the TD-DFT approach for $t=0$ have energies of $2.4,2.6$, and $2.7 \mathrm{eV}$. These transitions correspond to $s \rightarrow p$ excitations within the cage and depend only slightly on the parameter $t$ and thus on details of the cage geometry. The matrix elements of these transitions are similar and about twice as large as that for the intercage transition $E_{1}$. Taking into account that each cage is surrounded by eight other cages, the probability of the intercage transitions should be in fact about 4 times higher than that of the intracage ones. This is indeed observed experimentally [3].

The calculated electron levels with respect to the valence and conduction bands are shown in Fig. 3. The occupied state of the electron localized within a cage is about $4 \mathrm{eV}$ above the top of the valence band and about $2.5 \mathrm{eV}$ below the framework conduction band. This is a

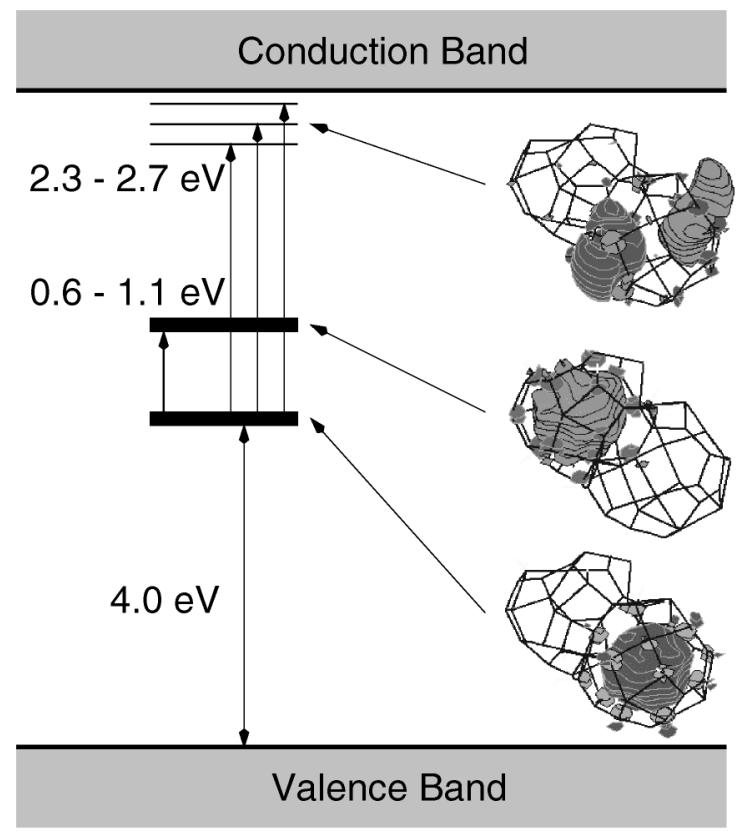

FIG. 3. Schematic of the energy levels of the system showing the optical transitions and character of electronic states. 
deep level, which agrees well with its localized character. The lowest unoccupied state is about $0.6-1.1 \mathrm{eV}$ higher and is localized in a neighboring cage. The shallow energy minima and the strong dependence of the first transition energy on the parameter $t$ suggest that the corresponding absorption band should be broadened with a maximum around $0.6 \mathrm{eV}$ and a tail towards larger energies. Other factors, such as disorder of the extra-framework oxygen ions, can also contribute to additional broadening. These results agree very well with the experimental data [3]. The second and several higher transitions have predominantly intracage character and the corresponding excited electronic states are very close to the bottom of the framework conduction band. This suggests that excitation in this band can lead to the electron ionization into the conduction band and thus to photoconductivity.

In summary, our calculations of the electronic structure and properties of a novel inorganic electride C12A7 show that electrons substituting anions in this material form deep electron states localized in positively charged cages. This differentiates it markedly from organic electrides and from many doped oxides having shallow donor states. The small barriers for hopping between cages, leading to high conductivity, are a reflection of a very flexible structure and the small distance between cages. On the other hand, these deep electron states, again very unusually, have two absorption bands: one in the infrared and another in UV. These we identify as intercage and intracage electron transitions, which determine the transparency gap of the material. Good agreement between theory and experiment supports the calculated electron gas properties. We predict that further increase of electron concentration by removing extra-framework oxygen ions should lead to interesting properties through electronelectron interaction and pairing, offering a rare opportunity to study a transition between different forms of electron localization. The results shed light on the be- havior of confined electron gas of different topology and electron-phonon coupling, and suggest new designs for new transparent conductors, electron emitters, and electrides.

We wish to thank J. L. Gavartin, I.V. Abarenkov, A. M. Stoneham, A.Y. Gal, and A. S. Foster for valuable discussions. This work is supported by Japan Science and Technology Corporation.

*Author to whom correspondence may be addressed. Electronic address: p.sushko@ucl.ac.uk

[1] A. S. Ichimura, J. L. Dye, M. A. Camblor, and L. A. Villaescusa, J. Am. Chem. Soc. 124, 1170 (2002).

[2] V. Petkov, S. J. L. Billinge, T. Vogt, A. S. Ichimura, and J. L. Dye, Phys. Rev. Lett. 89, 075502 (2002).

[3] K. Hayashi, S. Matsuishi, T. Kamiya, M. Hirano, and H. Hosono, Nature (London) 419, 462 (2002).

[4] S. Matsuishi, Y. Toda, M. Miyakawa, K. Hayashi, T. Kamiya, M. Hirano, I. Tanaka, and H. Hosono, Science 301, 626 (2003).

[5] D. J. Singh, H. Krakauer, C. Haas, and W. E. Pickett, Nature (London) 365, 39 (1993).

[6] H. Bartl and T. Scheller, Neues Jahrb. Mineral. Monatshefte 35, 547 (1970).

[7] A. Stein, G. A. Ozin, and G. D. Stucky, J. Am. Chem. Soc. 114, 8119 (1992).

[8] J. L. Dye, Science 247, 663 (1990).

[9] P.V. Sushko, A. L. Shluger, and C. R. A. Catlow, Surf. Sci. 450, 153 (2000).

[10] V. B. Sulimov, P.V. Sushko, A. H. Edwards, A. L. Shluger, and A. M. Stoneham, Phys. Rev. B 66, 024108 (2002).

[11] M. O. Zacate and R.W. Grimes, J. Phys. Chem. Solids 63, 675 (2002)

[12] W. R. Wadt and P. J. Hay, J. Chem. Phys. 82, 284 (1985).

[13] M. J. Frisch et al., GAUSSIAN98 (Gaussian Inc., Pittsburgh, PA, 1998).

[14] P.V. Sushko et al. (to be published). 